\title{
L-Citrulline supplementation attenuates blood pressure, wave reflection and arterial stiffness responses to metaboreflex and cold stress in overweight men
}

\author{
Arturo Figueroa $^{1 *}$, Stacey Alvarez-Alvarado ${ }^{1}$, Salvador J. Jaime ${ }^{1}$ and Roy Kalfon ${ }^{2}$ \\ ${ }^{1}$ Department of Nutrition, Food and Exercise Sciences, Florida State University, Tallahassee, FL 32306, USA \\ ${ }^{2}$ Department of Molecular Genetics, The Rappaport Family Institute for Research in the Medical Sciences, Technion - Israel \\ Institute of Technology, Haifa 31096, Israel
}

(Submitted 11 November 2015 - Submitted 24 March 2016 - Accepted 5 April 2016 - First published online 10 May 2016)

\section{Abstract}

Combined isometric exercise or metaboreflex activation (post-exercise muscle ischaemia (PEMI)) and cold pressor test (CPT) increase cardiac afterload, which may lead to adverse cardiovascular events. L-Citrulline supplementation (L-CIT) reduces systemic arterial stiffness (brachial-ankle pulse wave velocity (baPWV)) at rest and aortic haemodynamic responses to CPT. The aim of this study was to determine the effect of L-CIT on aortic haemodynamic and baPWV responses to PEMI + CPT. In all, sixteen healthy, overweight/obese males (age 24 (SEM 6) years; BMI 29.3 (SEM $4.0) \mathrm{kg} / \mathrm{m}^{2}$ ) were randomly assigned to placebo or L-CIT ( $\left.6 \mathrm{~g} / \mathrm{d}\right)$ for $14 \mathrm{~d}$ in a cross-over design. Brachial and aortic systolic blood pressure (SBP), diastolic blood pressure (DBP) and mean arterial pressure (MAP), aortic augmented pressure (AP), augmentation index (AIx), baPWV, reflection timing (Tr) and heart rate (HR) were evaluated at rest and during isometric handgrip exercise (IHG), PEMI and PEMI + CPT at baseline and after $14 \mathrm{~d}$. No significant effects were evident after L-CIT at rest. L-CIT attenuated the increases in aortic SBP and wave reflection (AP and AIx) during IHG, aortic DBP, MAP and AIx during PEMI, and aortic SBP, DBP, MAP, AP, AIx and baPWV during PEMI + CPT compared with placebo. HR and Tr were unaffected by L-CIT in all conditions. Our findings demonstrate that L-CIT attenuates aortic blood pressure and wave reflection responses to exercise-related metabolites. Moreover, L-CIT attenuates the exaggerated arterial stiffness response to combined metaboreflex activation and cold exposure, suggesting a protective effect against increased cardiac afterload during physical stress.

Key words: Metaboreflex activation: Cold pressor test: Isometric exercise: Arterial stiffness: Wave reflection: Aortic blood pressure: L-Citrulline supplementation

Excessive systolic blood pressure (SBP) reactivity to the cold pressor test (CPT), through increased sympathetic activity, may predict the development of hypertension in young, healthy adults $^{(1,2)}$. A greater sympathetic reactivity to the CPT has been demonstrated in overweight than in lean adults despite similar brachial blood pressure (BP) responses ${ }^{(2)}$. However, brachial BP has lower sensitivity to the $\mathrm{CPT}$ than aortic $\mathrm{BP}^{(3)}$. In young, normotensive adults, CPT increases aortic $\mathrm{BP}$, wave reflection and arterial stiffness (pulse wave velocity (PWV)) ${ }^{(4,5)}$. An increased BP reactivity to $\mathrm{CPT}$ may predict future increases in $\mathrm{PWV}^{(6)}$, which precedes the development of hypertension in obesity ${ }^{(7)}$.

Isometric handgrip exercise (IHG) also elicits a sympatheticmediated increase in $\mathrm{BP}^{(8)}$ known as the exercise pressor response. The $\mathrm{BP}$ response to IHG is determined by neural signals from the brain (central command) and active muscles (mechanoreceptors and metaboreceptors) ${ }^{(8,9)}$. Vascular responses to muscle metaboreflex activation can be isolated from central command and mechanoreceptor influences by trapping metabolites in the previously exercised muscle via arterial occlusion (post-exercise muscle ischaemia (PEMI) ${ }^{(8-10)}$. Exaggerated $\mathrm{BP}$ and vasoconstrictor responses to PEMI have been shown in young, obese adults with pre-hypertension ${ }^{(11)}$.

Acute increases in aortic haemodynamics and PWV are exaggerated during concurrent IHG and CPT (cold-pain stimulus) in young, healthy adults ${ }^{(5,12)}$. Similarly, we recently reported that PEMI with concurrent CPT (PEMI + CPT) caused greater increases in aortic haemodynamics and PWV than PEMI alone in young, healthy, overweight/obese men ${ }^{(13)}$. As the combined stimuli of exercise and cold may increase the risk for adverse cardiovascular events in susceptible individuals by elevating myocardial work $^{(14)}$, therapies targeted to attenuate cardiac afterload during stress would be cardioprotective.

L-Arginine is the substrate for endothelial nitric oxide (NO) production, a potent vasodilator. L-Citrulline supplementation

Abbreviations: AIx, augmentation index; AP, augmented pressure; baPWV, brachial-ankle pulse wave velocity; BP, blood pressure; CPT, cold pressor test; DBP, diastolic blood pressure; IHG, isometric handgrip exercise; L-CIT, L-citrulline supplementation; MAP, mean arterial pressure; NO, nitric Oxide; PEMI, post-exercise muscle ischaemia; PWV, pulse wave velocity; SBP, systolic blood pressure; $\mathrm{Tr}$, transit time of the reflected wave.

* Corresponding author: A. Figueroa, fax +1 850645 5000, email afiguero@fsu.edu 
(L-CIT) increases plasma L-arginine and NO levels more efficiently than L-arginine supplementation ${ }^{(15,16)}$. At rest, L-CIT has reduced $\mathrm{BP}$, wave reflection and PWV in middle-aged adults with increased BP and/or arterial stiffness ${ }^{(17,18)}$, but not in young, healthy men ${ }^{(4)}$. L-CIT, either synthetic or from watermelon, has attenuated aortic haemodynamic responses to CPT in young, normotensive ${ }^{(4)}$ and older, hypertensive adults ${ }^{(19)}$. Yet, the possible benefits of L-CIT on vascular reactivity during metaboreflex activation remain unknown.

The aim of this study was to determine the effects of L-CIT on aortic haemodynamics and PWV responses to stress in healthy, young men. We hypothesised that L-CIT would attenuate vascular reactivity to PEMI with and without CPT, although the benefits would be more apparent during combined stress.

\section{Methods \\ Study population}

In total, sixteen overweight/obese (BMI $>25$ and $<40 \mathrm{~kg} / \mathrm{m}^{2}$ ) healthy males (18-35 years) participated in this study. Participants were non-smokers, sedentary $(<120 \mathrm{~min} /$ week of exercise) and free of overt chronic diseases. Exclusion criteria included brachial systolic BP (SBP) $\geq 140 \mathrm{mmHg}$ and use of medications and/or any supplements that may affect outcome variables. Participants were asked to maintain their ordinary diet and exercise habits. The study was approved by the Institutional Human Subjects Committee, and participants gave their written informed consent before participation.

\section{Study design}

This was a randomised, double-blind, placebo-controlled study. Participants were familiarised with the study protocols. Cardiovascular parameters were evaluated in a quiet, temperaturecontrolled room $\left(22-24^{\circ} \mathrm{C}\right)$ after an overnight fast and abstinence from beverages containing caffeine or alcohol for $12 \mathrm{~h}$ and from intense or prolonged physical activity $24 \mathrm{~h}$ before testing.

After 15 min of supine rest, cardiovascular parameters were measured in duplicate and averaged at rest and during the last minute of IHG and PEMI without CPT. Following $20 \mathrm{~min}$ of recovery, cardiovascular parameter measurements were repeated at rest, IHG and PEMI $+\mathrm{CPT}$. The trial order was randomised. After baseline measurements, participants were randomly assigned to placebo (maltodextrin) or L-CIT for $14 \mathrm{~d}$, followed by a 14-d washout period, and were then crossed over to the other supplementation. Measurements were repeated $14 \mathrm{~d}$ after both periods.

\section{Measurements}

Anthropometrics. Body weight and height were measured using a Seca Scale and stadiometer (Sunbeam Products) to the nearest $0.1 \mathrm{~kg}$ and $0.01 \mathrm{~m}$, respectively. Waist circumference was measured using a tape measure to the nearest $0.5 \mathrm{~cm}$ at the superior border of the iliac crest.
Haemodynamics. An automated oscillometric device (HEM-705CP; Omron Healthcare) was used to record brachial BP. These values were used to calibrate radial pressure waveforms obtained from a $10 \mathrm{~s}$ epoch with a high-fidelity tonometer (SPT-301B; Millar Instruments). Aortic waveforms were derived from a generalised transfer function (SphygmoCor, AtCor Medical) and composed of forward (P1) and reflected (P2) waves. Augmented pressure $(\mathrm{AP}=\mathrm{P} 2-\mathrm{P} 1)$ expressed as a percentage of the aortic pulse pressure gives the augmentation index (AIx). Transit time of the reflected wave ( $\mathrm{Tr}$ ) indicates the round-trip travel of the forward wave to the peripheral reflecting sites and return to the aorta. Tr and AIx have been used as markers of aortic stiffness and wave reflection, respectively. The intraclass correlation coefficient for aortic haemodynamics in our laboratory calculated on two separate days was $\geq 0.94$.

Arterial stiffness. Following a 15-min rest period, PWV was evaluated using a device (VP-2000; Omron Healthcare) composed of BP cuffs positioned around the arms (brachial artery) and ankles (posterior tibial artery). Waveforms were measured simultaneously by cuff sensors, whereas the transient time was calculated automatically by relating the foot of the forward wave to the R-wave of the electrocardiogram. The distance between sampling points was calculated automatically according to the participant's height. Brachial-ankle pulse wave velocity (baPWV) was measured as the time it takes for the pulse wave to travel from the arm sensor to the ankle sensor, divided by the distance between the sensors $(\mathrm{m} / \mathrm{s})$. Heart rate $(\mathrm{HR})$ was measured from the electrocardiogram. baPWV was not measured during IHG because of the difficulty in collecting pulse waveforms in the exercising arm.

Exercise and metaboreflex activation. The highest of three maximal compressions using a handgrip dynamometer (Lafayette Instrument Co.) was considered the maximal voluntary contraction (MVC). IHG was performed with the dominant arm at $30 \%$ of MVC for 2 min using a handgrip dynamometer. Visual feedback and verbal encouragement were provided to maintain the targeted grip force; $10 \mathrm{~s}$ before completion of the IHG, a cuff positioned proximally on the exercising forearm was inflated to suprasystolic levels $(200 \mathrm{mmHg}$ or at least $50 \mathrm{mmHg}$ above the brachial SBP during IHG) for 3 min with an automated pneumatic device (Hokanson E20; Hokanson, Inc.).

Cold pressor test. The left foot of participants was passively immersed into ice-cold water $\left(4^{\circ} \mathrm{C}\right)$ for 3 min during the PEMI + CPT trial. Participants were instructed to maintain consistent breathing rate/depth throughout the test.

L-Citrulline supplementation. Participants were instructed to ingest four capsules of $750 \mathrm{mg}$ L-citrulline or placebo (NOW Foods) twice a day (before breakfast and sleep) for $14 \mathrm{~d}$. To avoid acute vascular effects of L-CIT, the last capsule was ingested $48 \mathrm{~h}$ before the 2-week testing. Participants returned unused capsules and records to assess their compliance. 
Statistical analyses. On the basis of previous data ${ }^{(4)}$, it was calculated that sixteen participants would provide $85 \%$ power (two-sided $\alpha=0.05$ ) to detect a $7.5 \%$ decrease in brachial SBP during PEMI + CPT after L-CIT. Statistical analyses were conducted using SPSS 21.0. Normality was confirmed by the Shapiro-Wilk test for all measurements. An independent $t$ test was used to assess possible between-group differences at baseline. Separate two-way repeated-measures ANOVA was performed to determine differences within and between interventions in cardiovascular parameters in the four conditions: rest, IHG, PEMI and PEMI + CPT. When a significant group-by-time interaction was identified, paired $t$ tests were used to assess within-group differences between baseline and 2 weeks. Data are presented as mean values with their standard errors. Statistical significance was set at $P<0.05$.

\section{Results}

Participant characteristics are presented in Table 1. Weight, BMI and waist circumference did not change significantly after both supplementations. Cardiovascular parameters before and after the supplementations are presented in Tables 2 and 3. There were no differences between supplementations at baseline and no significant changes were detected after 2 weeks.

During IHG, L-CIT decreased brachial SBP $(P<0 \cdot 05)$, diastolic blood pressure (DBP) $(P<0.05)$ and mean arterial pressure (MAP) $(P<0.05)$ as well as aortic SBP $(P<0.01)$, AP $(P<0.001)$ and AIx $(P<0.001)$ compared with the placebo group (group-by-time interactions $P<0 \cdot 05$ ). L-CIT had no effect on HR, aortic DBP, aortic MAP and Tr.

During isolated PEMI, L-CIT reduced brachial DBP $(P<0.05)$ and aortic DBP $(P<0.01)$, MAP $(P<0.05)$ and AIx $(P<0.001)$ compared with placebo (group-by-time interaction $P<0.05$ ). HR, brachial SBP and MAP, aortic SBP, AP, Tr and baPWV (Fig. 1) were not affected by L-CIT.

During PEMI + CPT, L-CIT decreased brachial SBP $(P<0 \cdot 01)$, DBP $(P<0.001)$ and MAP $(P<0.001)$ as well as aortic SBP $(P<0.001), \mathrm{DBP}(P<0.001), \mathrm{AP}(P<0.001), \mathrm{AIx}(P<0.001)$ and baPWV (Fig. $1, P<0.05$ ) compared with placebo (group-by-time interaction $P<0 \cdot 05$ ). L-CIT did not affect HR and Tr.

\section{Discussion}

The main findings of our study are that L-CIT attenuated (1) aortic BP and AIx responses to IHG, PEMI and PEMI + CPT and (2) baPWV response to PEMI + CPT but not to PEMI alone

Table 1. Study participant characteristics

(Mean values with their standard errors; $n$ 16)

\begin{tabular}{lrr}
\hline Variable & Mean & SEM \\
\hline Age (years) & 24.0 & $2 \cdot 0$ \\
Height $(\mathrm{m})$ & 1.7 & 0.0 \\
Body weight $(\mathrm{kg})$ & 86.8 & 3.7 \\
BMl $\left(\mathrm{kg} / \mathrm{m}^{2}\right)$ & 29.3 & 1.1 \\
Waist circumference $(\mathrm{cm})$ & 98.0 & 3.0 \\
\hline
\end{tabular}

in young, healthy, overweight/obese men. These findings suggest that L-CIT effectively buffered exercise- and coldinduced vascular reactivity.

L-CIT failed to reduce resting cardiovascular parameters in healthy, overweight/obese, young men. These findings are consistent with previous data showing unapparent effects of L-CIT on resting $\mathrm{BP}$ and wave reflection in young, normotensive, lean men due to normal BP and arterial function ${ }^{(4)}$. In contrast, Bailey et $a l .{ }^{(15)}$ recently reported a decrease in resting brachial $\mathrm{BP}$ in young, normotensive men after 1 week of L-CIT. It is possible that these results could reflect an acute vascular effect as L-citrulline was ingested $90 \mathrm{~min}$ before the BP measurement. Cardiovascular parameters were measured $48 \mathrm{~h}$ after the last L-citrulline dose to assess the chronic rather than the acute vascular effects. We have reported unapparent vascular effects of L-CIT in normotensive or pre-hypertensive individuals ${ }^{(4,20)}$. In contrast, L-CIT and watermelon supplementation have reduced resting $\mathrm{BP}$ in pre-hypertensive $^{(20)}$ and hypertensive adults $^{(18,19)}$ as well as resting baPWV in middle-aged and older adults ${ }^{(17,18)}$.

L-CIT attenuated brachial BP and wave reflection responses to IHG. Previous studies have reported that treatment with nebivolol, which exerts a vasodilator effect via endothelial NO production, reduced MAP responses to IHG in hypertensives ${ }^{(21)}$. These findings suggest that improved peripheral vasodilation attenuates the exercise pressor response. During PEMI following IHG, sympathetic-mediated peripheral vasoconstriction maintains SBP and MAP higher than at rest but similar to that during $\mathrm{IHG}^{(8,9)}$. In healthy, young men, the BP response to PEMI is attributed to increases in peripheral resistance $^{(9)}$ and stroke volume ${ }^{(22,23)}$. In the present study, IHG and PEMI elevated brachial SBP to 145 and $135 \mathrm{mmHg}$, respectively, at baseline. We found that L-CIT did not reduce SBP during PEMI, suggesting that L-CIT is less effective in reducing a small SBP response to PEMI in young, healthy men. Importantly, L-CIT reduced aortic DBP and wave reflection (AP and AIx) during PEMI, which may indicate a decreased vasomotor tone. Previous studies have demonstrated that acute NO-donor or antihypertensive therapy acutely decreases resting DBP and wave reflection magnitude independently of reflection timing (Tr) via reduction in vascular tone in the small arteries but not in the aorta ${ }^{(24,25)}$. Therefore, despite persistent elevation of sympathetic activation during PEMI ${ }^{(8)}$, L-CIT may counteract the increase in vasomotor tone during metaboreflex activation in young, healthy men.

Young, normotensive, overweight men have an exaggerated sympathetic reactivity to $\mathrm{CPT}^{(2)}$, which may be a mechanism for cold-induced hypertension ${ }^{(14)}$. The CPT evokes a sympathoexcitation-mediated hypertensive response through skin cooling and pain sensation ${ }^{(14)}$. Previous studies have shown that haemodynamic responses are greater during combined stimulation by IHG or PEMI with cold exposure compared with IHG or PEMI alone in young, healthy adults ${ }^{(5,12)}$. Similarly, we found greater haemodynamic response to PEMI + CPT compared with isolated PEMI in young men ${ }^{(13)}$.

We noted attenuation of haemodynamic responses to PEMI + CPT after L-CIT. Consistent with these findings, we previously reported reductions in aortic SBP and AIx responses to CPT or whole-body cold exposure following 2-4 weeks of 
Table 2. Haemodynamic parameters before and after supplementation (Mean values with their standard errors; $n$ 16)

\begin{tabular}{|c|c|c|c|c|c|c|c|c|c|c|}
\hline \multirow[b]{3}{*}{ Variables } & \multirow[b]{3}{*}{ Conditions } & \multicolumn{4}{|c|}{ Placebo (n 16) } & \multicolumn{4}{|c|}{ L-CIT $(n$ 16) } & \multirow[b]{3}{*}{ Group differences $†$} \\
\hline & & \multicolumn{2}{|c|}{ Before } & \multicolumn{2}{|c|}{ After } & \multicolumn{2}{|c|}{ Before } & \multicolumn{2}{|c|}{ After } & \\
\hline & & Mean & SEM & Mean & SEM & Mean & SEM & Mean & SEM & \\
\hline \multirow[t]{4}{*}{ Heart rate (beats/min) } & Rest & 58 & 1 & 59 & 2 & 58 & 2 & 60 & 2 & 0.51 \\
\hline & IHG & 70 & 3 & 76 & 3 & 74 & 3 & 72 & 3 & 0.03 \\
\hline & PEMI & 57 & 2 & 58 & 2 & 57 & 2 & 57 & 2 & 0.75 \\
\hline & PEMI + CPT & 67 & 3 & 65 & 2 & 66 & 3 & 67 & 2 & 0.39 \\
\hline \multirow[t]{4}{*}{ Brachial SBP $(\mathrm{mmHg})$} & Rest & 122 & 2 & 124 & 2 & 123 & 3 & 121 & 3 & 0.20 \\
\hline & IHG & 139 & 3 & 144 & 4 & 145 & 4 & $136^{\star}$ & 4 & 0.00 \\
\hline & PEMI & 132 & 2 & $138^{*}$ & 3 & 135 & 2 & 131 & 3 & 0.01 \\
\hline & $\mathrm{PEMI}+\mathrm{CPT}$ & 145 & 3 & 145 & 3 & 150 & 3 & $138^{\star \star}$ & 3 & 0.00 \\
\hline \multirow[t]{4}{*}{ Brachial DBP $(\mathrm{mmHg})$} & Rest & 67 & 1 & 69 & 1 & 68 & 2 & 64 & 2 & 0.03 \\
\hline & $\mathrm{IHG}$ & 76 & 2 & 79 & 3 & 76 & 3 & $65^{\star}$ & 4 & 0.00 \\
\hline & PEMI & 72 & 2 & 74 & 2 & 72 & 2 & $68^{*}$ & 2 & 0.01 \\
\hline & $\mathrm{PEMI}+\mathrm{CPT}$ & 80 & 2 & 80 & 2 & 88 & 2 & $72^{\star \star \star}$ & 2 & 0.00 \\
\hline \multirow[t]{4}{*}{ Brachial MAP $(\mathrm{mmHg})$} & Rest & 88 & 2 & 92 & 2 & 90 & 2 & 87 & 2 & 0.02 \\
\hline & $\mathrm{IHG}$ & 106 & 3 & 111 & 5 & 102 & 4 & $94^{*}$ & 4 & 0.01 \\
\hline & PEMI & 98 & 2 & 100 & 2 & 98 & 2 & 95 & 2 & 0.09 \\
\hline & PEMI + CPT & 107 & 2 & 105 & 2 & 109 & 2 & $96^{\star \star \star}$ & 2 & 0.00 \\
\hline \multirow[t]{4}{*}{ Aortic SBP $(\mathrm{mmHg})$} & Rest & 105 & 2 & 106 & 2 & 104 & 3 & 101 & 2 & 0.10 \\
\hline & IHG & 121 & 3 & 125 & 5 & 126 & 3 & $118^{\star \star}$ & 3 & 0.00 \\
\hline & PEMI & 117 & 3 & 121 & 4 & 120 & 3 & 116 & 4 & 0.07 \\
\hline & $\mathrm{PEMI}+\mathrm{CPT}$ & 127 & 4 & 129 & 4 & 132 & 4 & $119^{\star \star \star}$ & 4 & 0.00 \\
\hline \multirow[t]{4}{*}{ Aortic DBP $(\mathrm{mmHg})$} & Rest & 69 & 1 & 69 & 2 & 68 & 2 & 66 & 2 & 0.41 \\
\hline & $\mathrm{IHG}$ & 77 & 2 & 78 & 3 & 78 & 3 & 81 & 3 & 0.80 \\
\hline & PEMI & 75 & 2 & 75 & 2 & 77 & 2 & $71^{\star \star}$ & 2 & 0.04 \\
\hline & PEMI + CPT & 80 & 2 & 82 & 2 & 84 & 2 & $75^{\star \star *}$ & 3 & 0.00 \\
\hline \multirow[t]{4}{*}{ Aortic MAP (mmHg) } & Rest & 84 & 2 & 85 & 2 & 84 & 2 & 81 & 2 & 0.22 \\
\hline & $\mathrm{IHG}$ & 98 & 3 & 101 & 4 & 101 & 3 & 98 & 3 & 0.13 \\
\hline & PEMI & 94 & 3 & 95 & 2 & 96 & 2 & $89^{*}$ & 3 & 0.02 \\
\hline & PEMI + CPT & 102 & 3 & 103 & 3 & 105 & 3 & $95^{\star \star \star}$ & 3 & 0.00 \\
\hline
\end{tabular}

L-CIT, L-citrulline; IHG, isometric handgrip; PEMI, post-exercise muscle ischaemia; PEMI + CPT, PEMI with concurrent cold pressor test; SBP, systolic blood pressure; DBP, diastolic blood pressure; MAP, mean arterial pressure.

${ }^{*} P<0.05,{ }^{* *} P<0.01,{ }^{* * *} P<0.001 \mathrm{v}$. before.

$\dagger$ Group $\times$ time interaction from ANOVA.

Table 3. Wave reflection parameters before and after supplementation (Mean values with their standard errors; $n 16$ )

\begin{tabular}{|c|c|c|c|c|c|c|c|c|c|c|}
\hline \multirow[b]{3}{*}{ Variables } & \multirow[b]{3}{*}{ Conditions } & \multicolumn{4}{|c|}{ Placebo (n 16) } & \multicolumn{4}{|c|}{ L-CIT $(n$ 16) } & \multirow[b]{3}{*}{ Group differences } \\
\hline & & \multicolumn{2}{|c|}{ Before } & \multicolumn{2}{|c|}{ After } & \multicolumn{2}{|c|}{ Before } & \multicolumn{2}{|c|}{ After } & \\
\hline & & Mean & SEM & Mean & SEM & Mean & SEM & Mean & SEM & \\
\hline \multirow[t]{4}{*}{ AP $(\mathrm{mmHg})$} & Rest & 1.6 & 0.9 & $2 \cdot 3$ & 0.9 & $2 \cdot 4$ & 0.8 & $1 \cdot 7^{\star}$ & 1.0 & 0.01 \\
\hline & IHG & 7.5 & 1.1 & 7.4 & 1.2 & $10 \cdot 4$ & 1.8 & $2 \cdot 1^{\star \star \star}$ & 1.0 & 0.00 \\
\hline & PEMI & 7.9 & 1.3 & $10 \cdot 6$ & $2 \cdot 0$ & 9.4 & $2 \cdot 2$ & $6 \cdot 4$ & $2 \cdot 0$ & 0.04 \\
\hline & PEMI + CPT & 8.0 & $2 \cdot 2$ & 8.9 & $2 \cdot 1$ & $11 \cdot 1$ & $2 \cdot 0$ & $3 \cdot 8^{\star \star \star}$ & 1.5 & 0.01 \\
\hline \multirow[t]{4}{*}{ Alx (\%) } & Rest & $5 \cdot 8$ & $2 \cdot 3$ & $6 \cdot 1$ & $2 \cdot 7$ & 7.9 & 1.7 & 7.6 & $2 \cdot 0$ & 0.47 \\
\hline & $\mathrm{IHG}$ & $16 \cdot 1$ & $2 \cdot 2$ & $15 \cdot 1$ & $2 \cdot 0$ & 18.9 & $2 \cdot 7$ & $5 \cdot 3^{\star * *}$ & $2 \cdot 7$ & 0.00 \\
\hline & PEMI & 17.9 & $2 \cdot 2$ & 21.1 & 3.2 & $18 \cdot 4$ & 3.6 & $9.7^{\star \star \star}$ & 3.5 & 0.00 \\
\hline & PEMI + CPT & $14 \cdot 6$ & 3.7 & $17 \cdot 6$ & 3.8 & 20.8 & 3.4 & $7 \cdot 3^{\star \star \star}$ & 3.0 & 0.00 \\
\hline \multirow[t]{4}{*}{$\operatorname{Tr}(\mathrm{ms})$} & Rest & $164 \cdot 3$ & $6 \cdot 4$ & 171.4 & $5 \cdot 6$ & $170 \cdot 8$ & $6 \cdot 4$ & $170 \cdot 8$ & $5 \cdot 3$ & 0.47 \\
\hline & IHG & $155 \cdot 2$ & $5 \cdot 3$ & $148 \cdot 2$ & 3.6 & 154.6 & $6 \cdot 1$ & 156.9 & $4 \cdot 3$ & 0.37 \\
\hline & PEMI & 159.3 & 7.5 & 148.6 & $5 \cdot 8$ & 155.0 & 5.7 & $150 \cdot 6$ & 3.5 & 0.63 \\
\hline & PEMI + CPT & 149.7 & $5 \cdot 1$ & 144.2 & $2 \cdot 7$ & 149.9 & 4.7 & $152 \cdot 2$ & $2 \cdot 1$ & 0.31 \\
\hline
\end{tabular}

L-CIT, L-citrulline; AP, augmentation pressure; IHG, isometric handgrip; PEMI, post-exercise muscle ischaemia; PEMI + CPT, PEMI with concurrent cold pressor test; Alx, augmentation index; $T r$, transit time of the reflected wave.

${ }^{\star} P<0.05,{ }^{\star \star *} P<0.001 \mathrm{v}$. before.

$\dagger$ Group $\times$ time interaction from ANOVA.

L-CIT in young, healthy men ${ }^{(4)}$. Similarly, L-citrulline from watermelon attenuated CPT-induced increases in aortic BP and $\mathrm{AP}$, but not in AIx, in older, obese adults with hypertension. An explanation for this discrepancy could be that AP is a better index of wave reflection at rest ${ }^{(26)}$ and during PEMI ${ }^{(27)}$ in older adults $^{(26)}$. On the other hand, AIx is a reliable measure of 
(a)

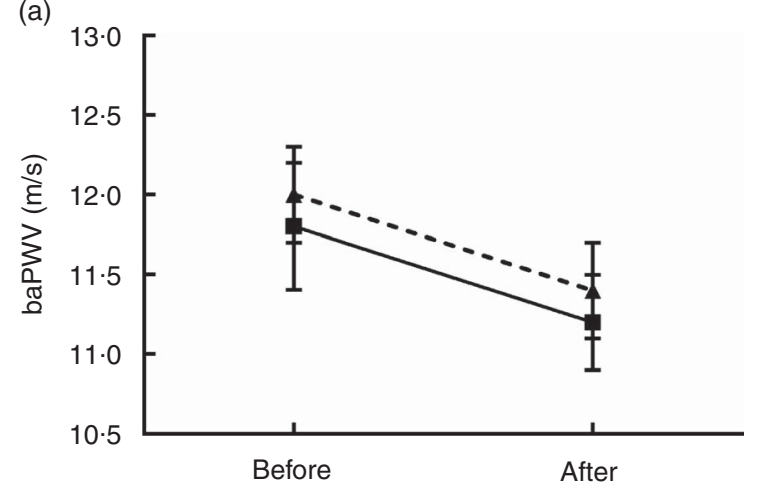

(b)

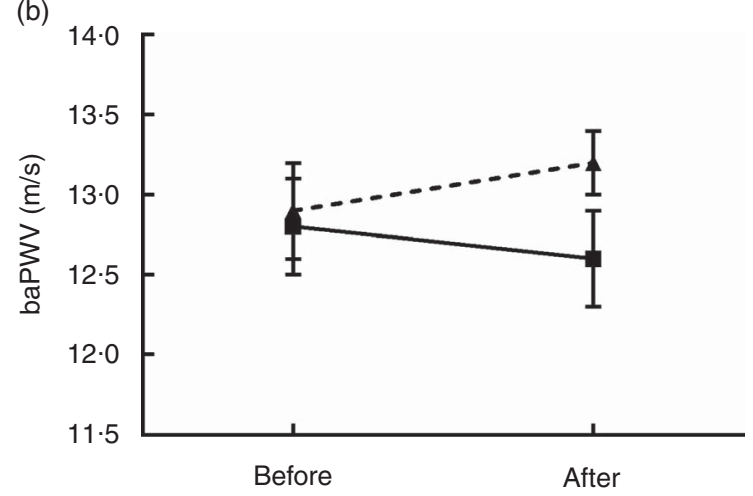

(c)

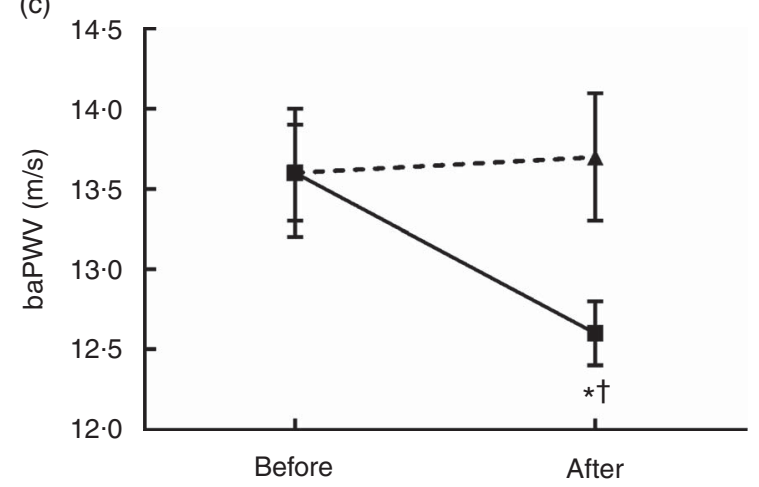

Fig. 1. Brachial-ankle pulse wave velocity (baPWV) at rest (a), during postexercise muscle ischaemia (PEMI) (b) and PEMI + cold pressor test (c) before and after placebo $\left(--\boldsymbol{\Delta}^{--}\right)$and L-citrulline $(-\boldsymbol{-})$ supplementation. Values are means with their standard errors. ${ }^{*} P<0.05 \mathrm{v}$. before; $\dagger P<0.05 \mathrm{v}$. placebo.

wave reflection in young adults ${ }^{(26)}$. Collectively, these findings indicate that L-CIT reduces cardiac afterload during cold exposure ${ }^{(19,28)}$, and our present findings add to the existing knowledge that L-CIT has a cardioprotective effect during concurrent PEMI and cold exposure. Our findings may be important for the prevention of adverse cardiac events in individuals with increased cardiovascular risk, as cold stimulus combined with exercise, but not cold alone, may increase the risk of myocardial ischaemia due to excessive cardiac afterload ${ }^{(14)}$.

Previous studies have shown that IHG or PEMI with concurrent CPT increases PWV by $1.2-1.8 \mathrm{~m} / \mathrm{s}$ in young men ${ }^{(5,13)}$. In the present study, L-CIT attenuated the baPWV response to PEMI + CPT by $1.06 \mathrm{~m} / \mathrm{s}$, whereas L-CIT was ineffective in reducing baPWV during isolated PEMI. At baseline, baPWV levels during PEMI with and without CPT were 13.6 and $12.8 \mathrm{~m} / \mathrm{s}$, respectively. Subsequently, L-CIT reduced baPWV during PEMI + CPT to relatively the same absolute level as that evoked during isolated PEMI. To the best of our knowledge, the efficacy of L-CIT on the PWV response to cold exposure has not been previously examined. A previous investigation has reported that the anti-stiffening effect of L-CIT on resting baPWV without cold exposure was attributed to an increased NO availability ${ }^{(17)}$; 1-week L-CIT and 6-week watermelon supplementation reduced baPWV by $1.2-1.3 \mathrm{~m} / \mathrm{s}$ in middle-aged adults with a baseline baPWV $>14 \mathrm{~m} / \mathrm{s}^{(17,18)}$. In the present study, only PEMI + CPT elevated baPWV close to this level, suggesting that L-CIT is able to exert a beneficial effect in conditions with increased PWV. Cold exposure ${ }^{(29)}$ and vasoconstrictor drugs (angiotensin II) ${ }^{(24)}$ predominantly increase peripheral (brachial) PWV. It is well known that femoral-ankle PWV (faPWV) is the largest peripheral arterial segment in baPWV ${ }^{(30)}$ and is increased by PEMI ${ }^{(10)}$. As Tr, an estimate index of aortic stiffness, was not affected by L-CIT, our findings suggest that L-CIT may have reduced faPWV compared with carotid-femoral PWV (cfPWV).

The present findings could be important for individuals with hypertension and heart failure, as they have an exaggerated sympathetically mediated haemodynamic response to exercise due to metaboreflex overactivation ${ }^{(8,31)}$. In heart failure patients, PEMI-induced increase in BP may lead to reduced exercise tolerance due to increased myocardial work ${ }^{(31)}$.

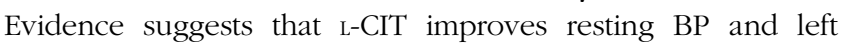
ventricular function in heart failure patients ${ }^{(32)}$. Future studies are needed to examine the effects of L-CIT on haemodynamic responses to exercise and PEMI in individuals with metaboreflex overactivation.

The exact mechanism by which L-CIT counteracts vascular reactivity to physical stress is unclear. Recent evidence supports that L-citrulline is a NO precursor, as its conversion to L-arginine leads to endothelial NO synthesis ${ }^{(15,16)}$. In young men, the increase in MAP during PEMI following IHG may have been secondary to vasoconstriction and not to increase in stroke volume $^{(9)}$. BP and AIx reductions by vasodilator drugs including NO donors is through a decrease in vascular smooth muscle tone of the peripheral arteries ${ }^{(24,25)}$. Although PWV is influenced by distending pressure (MAP), changes in PWV lead to changes in $\mathrm{BP}^{(7)}$. Therefore, attenuated peripheral vasoconstriction during physical stress may account for our findings.

Our study is limited by the lack of measurement of stroke volume and plasma L-arginine and NO levels. We did not measure the main components of baPWV (cfPWV and faPWV) because of technical limitations (hip was passively flexed to submerge the foot in cold water). However, baPWV is highly correlated with cfPWV ${ }^{(30)}$, and increased values of both indices are similarly associated with cardiovascular risk factors ${ }^{(33)}$. Our participants were young, healthy men and the present findings may not be translated to other populations. Finally, the multiple comparisons performed in our study are a statistical concern because they may lead to false-positive significant findings.

In conclusion, our findings demonstrate that 2-week L-CIT attenuated aortic BP, wave reflection and systemic arterial stiffness responses to stress induced by exercise-related 
metabolites and local pain/cold exposure. In addition, the vascular protective effect of L-CIT during metaboreflex activation was more pronounced during cold exposure.

\section{Acknowledgements}

The authors thank NOW foods for providing the L-citrulline and placebo capsules without charge. NOW foods had no role in the design, analysis or writing of this article.

This research received no specific grant from any funding agency, commercial or not-for-profit sectors.

The contributions of the authors are as follows: A. F. and R. K. designed the study and analysed the data. R. K., S. A.-A. and S. J. J. collected the data. A. F. wrote the manuscript. All the authors reviewed and approved the final version of the manuscript. A. F. had the primary responsibility of the study.

None of the authors has any conflicts of interest to declare.

\section{References}

1. Menkes MS, Matthews KA, Krantz DS, et al. (1989) Cardiovascular reactivity to the cold pressor test as a predictor of hypertension. Hypertension 14, 524-530.

2. Park J, Middlekauff HR \& Campese VM (2012) Abnormal sympathetic reactivity to the cold pressor test in overweight humans. Am J Hypertens 25, 1236-1241.

3. Edwards DG, Gauthier AL, Hayman MA, et al. (2006) Acute effects of cold exposure on central aortic wave reflection. J Appl Physiol 100, 1210-1214.

4. Figueroa A, Trivino JA, Sanchez-Gonzalez MA, et al. (2010) Oral L-citrulline supplementation attenuates blood pressure response to cold pressor test in young men. Am J Hypertens 23, $12-16$.

5. Geleris P, Stavrati A \& Boudoulas H (2004) Effect of cold, isometric exercise, and combination of both on aortic pulse in healthy subjects. Am J Cardiol 93, 265-267.

6. Bellinazzi VR, Sposito AC, Schreiber R, et al. (2014) Response to cold pressor test predicts long-term changes in pulse wave velocity in men. Am J Hypertens 27, 157-161.

7. Weisbrod RM, Shiang T, Al Sayah L, et al. (2013) Arterial stiffening precedes systolic hypertension in diet-induced obesity. Hypertension 62, 1105-1110.

8. Delaney EP, Greaney JL, Edwards DG, et al. (2010) Exaggerated sympathetic and pressor responses to handgrip exercise in older hypertensive humans: role of the muscle metaboreflex. $A m \mathrm{~J}$ Physiol Heart Circ Physiol 299, H1318-H1327.

9. McNulty CL, Moody WE, Wagenmakers AJM, et al. (2014) Effect of muscle metaboreflex activation on central hemodynamics and cardiac function in humans. Appl Physiol Nutr Metab 39, 861-870.

10. Davies TS, Frenneaux MP, Campbell RI, et al. (2007) Human arterial responses to isometric exercise: the role of the muscle metaboreflex. Clin Sci (Lond) 112, 441-447.

11. da Silva RP, Martinez D, Faria CC, et al. (2013) Improvement of exercise capacity and peripheral metaboreflex after bariatric surgery. Obes Surg 23, 1835-1841.

12. Koutnik AP, Figueroa A, Wong A, et al. (2014) Impact of acute whole-body cold exposure with concurrent isometric handgrip exercise on aortic pressure waveform characteristics. Eur J Appl Physiol 114, 1779-1787.

13. Kalfon R, Campbell J, Alvarez-Alvarado S, et al. (2015) Aortic hemodynamics and arterial stiffness responses to muscle metaboreflex activation with concurrent cold pressor test. $\mathrm{Am}$ J Hypertens 28, 1332-1338.

14. Manou-Stathopoulou V, Goodwin CD, Patterson $\mathrm{T}$, et al. (2015) The effects of cold and exercise on the cardiovascular system. Heart 101, 808-820.

15. Bailey SJ, Blackwell JR, Lord T, et al. (2015) L-citrulline supplementation improves $\mathrm{O}_{2}$ uptake kinetics and high-intensity exercise performance in humans. J Appl Physiol 119, 385-395.

16. Morita M, Hayashi T, Ochiai M, et al. (2014) Oral supplementation with a combination of L-citrulline and L-arginine rapidly increases plasma L-arginine concentration and enhances NO bioavailability. Biochem Biophys Res Commun 454, 53-57.

17. Ochiai M, Hayashi T, Morita M, et al. (2012) Short-term effects of L-citrulline supplementation on arterial stiffness in middleaged men. Int J Cardiol 155, 257-261.

18. Figueroa A, Wong A, Hooshmand S, et al. (2013) Effects of watermelon supplementation on arterial stiffness and wave reflection amplitude in postmenopausal women. Menopause 20, 573-577.

19. Figueroa A, Wong A \& Kalfon R (2014) Effects of watermelon supplementation on aortic hemodynamic responses to the cold pressor test in obese hypertensive adults. Am J Hypertens 27, 899-906.

20. Figueroa A, Sanchez-Gonzalez MA, Perkins-Veazie PM, et al. (2011) Effects of watermelon supplementation on aortic blood pressure and wave reflection in individuals with prehypertension: a pilot study. Am J Hypertens 24, 40-44.

21. Price A, Raheja P, Wang Z, et al. (2013) Differential effects of nebivolol versus metoprolol on functional sympatholysis in hypertensive humans. Hypertension 61, 1263-1269.

22. Crisafulli A, Scott AC, Wensel R, et al. (2003) Muscle metaboreflex-induced increases in stroke volume. Med Sci Sports Exerc 35, 221-228.

23. Figueroa A, Hooshmand S, Figueroa M, et al. (2010) Cardiovagal baroreflex and aortic hemodynamic responses to isometric exercise and post-exercise muscle ischemia in resistance trained men. Scand J Med Sci Sports 20, 305-309.

24. Kelly RP, Millasseau SC, Ritter JM, et al. (2001) Vasoactive drugs influence aortic augmentation index independently of pulse-wave velocity in healthy men. Hypertension 37, $1429-1433$

25. Hashimoto J, Westerhof BE, Westerhof $\mathrm{N}$, et al. (2008) Different role of wave reflection magnitude and timing on left ventricular mass reduction during antihypertensive treatment. J Hypertens 26, 1017-1024.

26. Fantin F, Mattocks A, Bulpitt CJ, et al. (2006) Is augmentation index a good measure of vascular stiffness in the elderly? Age Ageing 36, 43-48.

27. Figueroa A, Jaime SJ, Johnson SA, et al. (2015) Impact of age on aortic wave reflection responses to metaboreflex activation and its relationship with leg lean mass in postmenopausal women. Exp Gerontol 70, 119-124.

28. Sanchez-Gonzalez MA, Koutnik AP, Ramirez K, et al. (2013) The effects of short term L-citrulline supplementation on wave reflection responses to cold exposure with concurrent isometric exercise. Am J Hypertens 26, 518-526.

29. King SG, Ahuja KDK, Wass J, et al. (2013) Effect of wholebody mild-cold exposure on arterial stiffness and central haemodynamics: a randomised, cross-over trial in healthy men and women. Eur J Appl Physiol 113, 1257-1269.

30. Sugawara J, Hayashi K, Yokoi T, et al. (2005) Brachial-ankle pulse wave velocity: an index of central arterial stiffness? J Hum Hypertens 19, 401-406. 
31. Keller-Ross ML, Johnson BD, Joyner MJ, et al. (2014) Influence of the metaboreflex on arterial blood pressure in heart failure patients. Am Heart J 167, 521-528.

32. Orozco-Gutiérrez JJ, Castillo-Martínez L, Orea-Tejeda A, et al. (2010) Effect of L-arginine or L-citrulline oral supplementation on blood pressure and right ventricular function in heart failure patients with preserved ejection fraction. Cardiol J 17, 612-618.

33. Yamashina A, Tomiyama H, Arai T, et al. (2003) Brachialankle pulse wave velocity as a marker of atherosclerotic vascular damage and cardiovascular risk. Hypertens Res 26, 615-622. 